\title{
ANÁLISIS DE LA CALIDAD DE VIDA LABORAL DE LAS PEQUEÑAS Y MEDIANAS EMPRESAS DEL SUR SONORA, A TRAVÉS DE REDES NEURONALES ARTIFICIALES
}

\author{
ANALYSIS OF THE QUALITY OF WORK LIFE OF SMALL AND MEDIUM-SIZED \\ COMPANIES IN SOUTHERN SONORA, THROUGH ARTIFICIAL NEURONAL \\ NETWORKS
}

\author{
Celia Yaneth Quiroz-Campas ${ }^{1} *$ (D); Francisco Espinoza-Morales ${ }^{2}$ (D) . \\ 1. Instituto Tecnológico de Sonora, México. celia.quiroz@itson.edu.mx \\ 2. Universidad de Sonora, México \\ *Autor de correspondencia: Celia Yaneth Quiroz-Campas, email: quirozcampasceliayaneth@gmail.com
}

\section{RESUMEN}

En esta investigación se realizó un análisis de Red neuronal artificial para 356 pequeñas y medianas empresas del Sur de Sonora, las Pymes representan el 99.8\% de las unidades económicas del país con más de 4.1 millones de empresas, según datos oficiales del Inegi este tipo de empresas que aportan $42 \%$ del Producto Interno Bruto (PIB) y generan $78 \%$ del empleo.

Para determinar su calidad de vida laboral se utilizó un instrumento donde se evaluaron a las empresas en cuatro dimensiones: bienestar individual, condiciones y medio ambiente de trabajo, organización y bienestar logrado a través del trabajo. Los resultados obtenidos mostraron una calidad de vida laboral del $85.25 \%$, con esta metodología aplicada demuestra la relevancia del estudio aplicable a nivel nacional e internacional.

Palabras clave: Calidad de vida laboral; trabajadores; red neuronal artificial; empresas.

Cómo citar:

Quiroz-Campas, Celia Yaneth; Espinoza-Morales, Francisco. (2021). Análisis de la calidad de vida laboral de las pequeñas y medianas empresas del sur sonora, a través de redes neuronales artificiales. Revista de Investigaciones Universidad del Quindio, 33(2), 127-137. https://doi.org/10.33975/riuq.vol33n2.746 


\section{ABSTRACT}

In this research, an artificial neural network analysis was carried out for 356 small and medium-sized companies in the South of Sonora, SMEs represent $99.8 \%$ of the economic units of the country with more than 4.1 million companies, according to official data from Inegi this type of companies that contribute $42 \%$ of the Gross Domestic Product (GDP) and generate $78 \%$ of employment.

To determine their quality of working life, an instrument was used where companies were evaluated in four dimensions: individual well-being, working conditions and environment, organization and well-being achieved through work. The results obtained showed a quality of work life of $85.25 \%$, with this applied methodology it demonstrates the relevance of the study applicable at the national and international level.

Keywords: Quality of work life; workers; artificial neural network; companies.

\section{INTRODUCCIÓN}

El objetivo de la presente investigación es realizar un análisis de la calidad de vida laboral de las pequeñas y medianas empresas del Sur Sonora, a través de redes neuronales artificiales, para encontrar áreas de oportunidad que nos permita seguir haciendo este tipo de estudios a corto, mediano y largo plazo.

Para Zapata \& Jara (2014) la calidad de vida laboral infiere en la salud, satisfacción y el bienestar de los trabajadores, y todo lo relacionado a su ambiente de trabajo; este tema tiene relación con aspectos laborales, que está enfocado a experiencias de cada trabajador, los objetivos y metas que persiguen las empresas. Por ello este es un concepto multidimensional, ya que está formado por objetivos y subjetivos, estos se enfocan en como los individuos se desenvuelven en su ambiente de trabajo.

De acuerdo con Urzúa y Caqueo (2012) la calidad de vida laboral se enfoca en todas las condiciones productivas e interpersonales, influyen de manera directa en cómo se desenvuelven psicológicamente los trabajadores en su ambiente laboral, en conjunto con la contribución a la manera en que los individuos se adaptan, la creatividad con la que cuentan y la voluntad que exigen los objetivos de la productividad de las instituciones.
De acuerdo con la Organización Mundial de la Salud (OMS), los empleados representan la mitad de la población en todo el mundo y son quienes aportan en mayor medida al desarrollo económico y social de muchos países, por lo tanto, su salud es importante, principalmente cuando está condicionada por riesgos del lugar de trabajo (OMS, 2009). En lo anterior mencionado, es importante tomar en cuenta que según estadísticas de la Organización Internacional del Trabajo (OIT) y de la OMS (2009), las condiciones de trabajo para la mayoría de los trabajadores en todo el mundo no reúnen los estándares y guías mínimos establecidos por estas dependencias para la salud ocupacional, la seguridad y la protección social. Además, es muy importante destacar que hay una inadecuada atención en materia de salud y seguridad ocupacional, lo que ocasiona que existan millones de muertes anualmente relacionadas con el trabajo, accidentes y enfermedades temporales. Todo esto ocasiona que la calidad de vida laboral de las personas este muy baja y que los trabajadores se sientas insatisfechos e inseguros en su trabajo.

Cardona \& Higuita (2014) realizaron un estudio donde los resultados obtenidos en su investigación permiten concluir lo siguiente: La sobrecarga en el trabajo y los bajos nivel de seguridad tienen un alto efecto en 2 factores: agotamiento emocional e insatisfacción de logro, que impactan directamente en la calidad de vida 
de los trabajadores y en su satisfacción laboral, disminuyendo su desempeño y la productividad de la empresa, convirtiéndolos en esclavos de su empleo y realizando sus actividades con una actitud negativa

Estudios realizados con enfoque a la calidad de vida laboral, indican una baja percepción de la misma, en conjunto con una baja motivación, lo que afecta en las fortalezas y en las debilidades del contexto laboral. Existe evidencia de que en las organizaciones se encuentra la sobrecarga laboral, un salario injusto, y poco tiempo disponible, que afecta de una manera desfavorable a las organizaciones, ya que no hay una sensación de bienestar por parte de los empleados (Zapata \& Jara, 2015).

Esto influye de manera directa en el rendimiento, su estado de ánimo, que puede relacionarse con altos niveles de ansiedad, los cuales interfieren en la capacidad que tienen los trabajadores para adaptarse a distintas exigencias en el trabajo. Se considera que las condiciones organizacionales y relacionales en los cuales se desenvuelve una ocupación resultan de manera crucial para que se identifique y se aborden las variables organizaciones y también las individuales que ayudan al bienestar subjetivo de los empleados.

\section{FUNDAMENTACIÓN TEÓRICA}

Según Manríquez \& Salazar (2015) la calidad de vida (CDV) se entiende como la idea que tienen las personas de su situación en la vida, en un contexto cultural, de valores y en relación con sus metas y objetivos, que es lo que esperan de algo el particular y el interés que se tiene sobre ello. Este concepto tiene componentes objetivos y subjetivos, los objetivos son condiciones externas como nivel de ingreso, vivienda, los servicios de salud, entre otros, mientras que los subjetivos se refieren a la calidad de vida y liderazgo.

Genéricamente, las pocas investigaciones que existen que tienen relación con el liderazgo, con la calidad de vida y la salud, se enfocan en la influencia del líder sobre la calidad de vida, la salud y en general, el bienestar de los trabajadores. Así, se ha ilustrado que el liderazgo que fomenta la autogestión, interviene de una manera positiva en la calidad de vida laboral y en la satisfacción de los trabajadores en las empresas (Juárez \& Contreras, 2012).

Según Manríquez \& Salazar (2015) las naciones cuentan con legislaciones laborales, leyes de seguridad social, vivienda y apoyos para el retiro, para cuidar a los trabajadores. Sin embargo, a nivel mundial hay una crisis económica esto hace que las organizaciones y el gobierno violen esas leyes, perjudicando al capital humano y deteriorando su calidad de vida. Es por ello que la ética tiene un papel de gran importancia que ha llevado al plano de las organizaciones, indudablemente está presente en la calidad laboral que el empresario ofrece a sus empleados, este es un tema de suma importancia, ya que el elemento humano es lo más importante que debe cuidar cualquier empresa, es cabalmente al que no se le da un valor justo, las estrategias de negocios son elaboradas para que se beneficie la empresa sin valorar muchas veces el beneficio para el empleado.

De acuerdo con Huerta, Contreras \& Almodóvar (2011) la calidad laboral debe basarse en el respeto, la confianza y la comunicación en la relación empresa-empleado. Es por eso que es considerada como un elemento de responsabilidad social en la parte interna de las empresas con el propósito de favorecer a un buen ambiente de trabajo adecuado y seguro, para que sus trabajadores sean creativo, innovadores y productivos. Con este hecho, la empresa se verá beneficiada ya que con esto se disminuye el ausentismo, rotación del personal y aumenta la productividad. El libro verde dice que la contratación no debe ser discriminatoria y que las organizaciones tienen que tener un mayor esfuerzo por mejorar la seguridad en el ambiente de trabajo. 
Para Granados (2014) los científicos de la administración se enfocaban en la eficiencia y la especialización de tareas en estructuras obsoletas y tradicionales de la empresa. Cuando esta estructura fue creciendo, lo que se buscaba era separar las tareas, buscando con ello la eficiencia, los bajos costos, y contratar empleados que no estuvieran calificados para que se capacitaran y pudieran aprender en poco tiempo para que lograran un buen desempeño laboral. Las tareas las definían de acuerdo a la jerarquía.

Para Lizarazo, Fajardo \& Quintana (2014) el concepto de calidad de vida laboral se creó en una serie de conferencias a 1 final de los años 60 y comienzos de los 70 por el Ministerio de Trabajo de los EE.UU y la fundación FORD. Dichas conferencias se estimularon por el fenómeno de la "alienación del trabajador" simbolizando por huelgas entre la población activa de los cuales la mayor para de esta eran jóvenes de la nueva planta de monta de la General Motors.

Se decía por las personas que asistían a las conferencias que el concepto de calidad de vida laboral estaba por encima de la satisfacción de cada puesto, esto incluía la participación por parte de los empleados en por lo menos algunos momentos de tomar decisiones, aumento de la autonomía en la jornada diaria, rediseñar los puestos de trabajo y estructuras de la empresa con el propósito de estimular el aprendizaje, y una manera satisfactoria de interés y participación en el trabajo, para que los empleados sintieran la inclusión y esto les proporcionara seguridad y bienestar dentro de la organización.

Según Carrasquel (2016) la Calidad de vida laboral fue reconocida social e institucionalmente gracias al movimiento de CVL (calidad de vida laboral). De este movimiento nace la necesidad de que las empresas sean más humanizadas en el ambiente laboral, poniendo énfasis en el desarrollo del factor humano y a mejorar la calidad de vida de los mismos. Desde ese momento el tema de calidad de vida se extendió tanto es EE.UU. como en Europa
A partir de este momento, el tema de la calidad de vida laboral se populariza tanto en los EE.UU. como en Europa. Al estudiar la calidad de vida laboral se ha abordado bajo dos perspectivas teórico-metodológicas: La calidad de vida en el trabajo y la perspectiva de la calidad de vida psicológica

Martínez, Oviedo \& Luna (2013) en los últimos años el concepto de calidad de vida ha tenido un gran lugar en todo el mundo, llamando la atención de los trabajadores y de los empresarios, a causa de esto se han creado distintos enfoques, que añaden a la común preocupación por la competitividad y productividad económica un énfasis en el cuidado de los empleados, así como en la importancia que tienen las áreas de trabajo y la seguridad que estas les aportan, además las organizaciones están al pendiente del estado de ánimo de los trabajadores, les interesa saber si se sientes motivados y principalmente si se sientes identificados con la empresa y con lo que hacen.

En general, la investigación de cómo son las condiciones de trabajo es complejo ya que se abordan temas que se encuentran fuera del ambiente laboral como: económicos, sociales, políticos, tecnológicos, ergonómicos, entre otros, en consecuencia la empresa tiene que trabajar en factores básicos que hacen sentir al empleado bien consigo mismo y con la organización por ejemplo: en las dimensiones básicas relativas al ambiente físico y social, a la organización, características de las tareas, a la salud y la seguridad, entre otras. Existen 3 factores que condicionan el trabajo de las personas y que impactan directamente en la calidad de vida laboral. Los factores se dividen en:

1. Condiciones Ambientales: incluye términos como la satisfacción laboral, los riegos laborales, la carga de trabajo, entre otros.

2. Condiciones Ergonómicas: estánrelacionadas con el lugar de trabajo donde el empleado realiza sus actividades y la seguridad que este le ofrece.

3. Condiciones Económicas: se refiere a la 
percepción que tiene el empleado de su trabajado con respecto al salario recibido.

En el mundo laboral, el factor primordial de la calidad en el producto o servicio ofrecido lo conforman las personas que trabajan en las organizaciones, y este está representado por la manera en la cual el empleado se siente satisfecho por el trabajo que realiza. Investigar y medir la calidad de vida en las empresas se ha transformado en una gran necesidad para lograr la excelencia. Algunos factores significativos para la calidad de vida de los empleados son: realización profesional, sobrecarga laboral, relación con los compañeros, medio físico, tecnología, horario y vida personal. Además, se incluyen factores como la conciliación de vida laboral y familiar, implicaciones laborales y estado de ánimo (Martínez, Oviedo \& Luna, 2013).

\section{METODOLOGÍA}

En esta investigación se integran las técnicas de redes neuronales artificiales y análisis de datos factoriales para evaluar la calidad de vida laboral de 356 pequeñas y medianas empresas del Sur de Sonora.

El instrumento utilizado fue diseñado por Hernández, Lumbreras, Méndez, Rojas, Cervantes \& Juárez (2017) integrado en cuatro dimensiones: 1) catorce ítems relacionados con la dimensión de "bienestar individual", la cual refleja aspectos del entorno de trabajo, la satisfacción laboral, motivación, expectativas, actitudes y valores; 2) doce ítems para "condiciones y medio ambiente de trabajo", que incluye condiciones, características, contenido de trabajo, seguridad e higiene; 3) catorce ítems para "organización", definida como la estructura de las relaciones y 4) quince ítems se relacionan con la dimensión "bienestar logrado a través del trabajo".

Por el origen de la investigación el método que se empleó fue de una forma deductiva, partiendo de un procedimiento general con el cual se llegará a distintos planteamientos. Los métodos específicos a desarrollar van a partir de un área de conocimiento de cada competencia, estos son definidos por el investigador.

En este apartado se presenta de una manera genérica un bosquejo de la metodología con la que realizo la investigación. El tipo de investigación es cuantitativa, (Hernández, Fernández y Baptista, 2014), debido a que se elaboraron preguntas de investigación e indicadores. Este estudio tiene como alcance el estudio exploratorio, porque se tiene como propósito medir la relación que existe entre las variables. Es un estudio teórico empírico, es empírico porque se rige en la experiencia del investigador, $\mathrm{y}$ es un modelo teórico porque se cuenta con información propia y se fundamenta con otras. La presente investigación tiene un diseño no experimental, porque se basa fundamentalmente en la observación de fenómenos tal y como se dan en su contexto natural, para después analizarlo. Es transaccional porque se recolectan datos en un solo momento, en un tiempo único.

La validez de contenido de los ítems propuestos para CVL-HP global presentó una concordancia entre los expertos de 0.834 (Kappa de Cohen). La validez de constructo se estableció con análisis factorial, integrado en cuatro dimensiones. Se evaluaron tres tipos de validez del instrumento: contenido, criterio y constructo. La primera se realizó mediante la construcción de un instrumento que mide cuatro dimensiones de la CVL-HP: "bienestar individual", "condiciones y medio ambiente de trabajo", "organización", y "bienestar logrado a través del trabajo". Una vez elaborado el cuestionario, éste se distribuyó a un grupo de psicólogos organizacionales, solicitándoles que validaran con base en los cinco criterios de Moriyama: compresión de los ítems, aplicabilidad, justificación, definición clara y factibilidad; donde cada criterio se calificó en escala de nada $=0$ a mucho $=3$.

La validez de criterio quedó establecida a través de la comparación de la escala propuesta CVL- 
HP con otro instrumento que mide la Calidad de Vida en el Trabajo, CVT-Gohisalo. La validez de constructo se evaluó mediante análisis factorial exploratorio, utilizando el método de componentes principales y con rotación simple varimax. La confiabilidad se midió utilizando el coeficiente Alfa de Cronbach.

Para evaluar la validez de contenido se determinó la concordancia con la prueba Kappa de Cohen. Posteriormente se realizó análisis factorial exploratorio al conjunto de 57 reactivos relacionados con la CVL para descubrir los factores subyacentes y la carga factorial de cada ítem.
Tabla 1. Estadísticas de fiabilidad

\begin{tabular}{cc}
\hline Alfa de Cronbach & N de elementos \\
\hline .880 & 55 \\
\hline Fuente: Elaboración propia
\end{tabular}

En la presente tabla se observa que el instrumento aplicado tiene una fiabilidad de .880

Según Gonzales (2015) el alfa de Cronbach debe estar entre 0.70 y 0.90 para que indique una consistencia interna buena para la escala unidimensional.

A continuación, se muestran los datos generales del sujeto de estudio.

Tabla 2. Datos Generales del instrumento aplicado

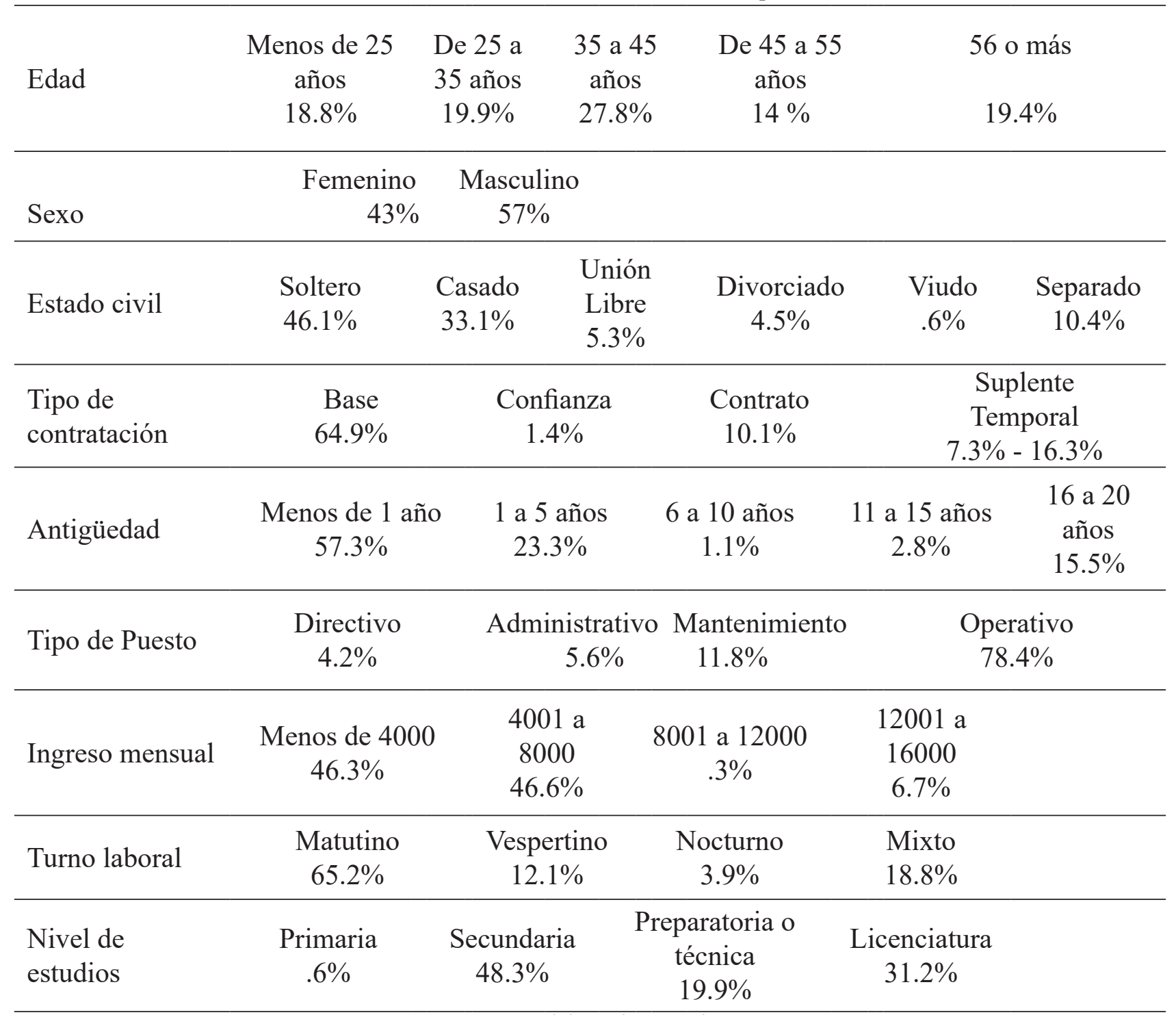




\section{RESULTADOS Y DISCUSIÓN}

En la tabla 3 se aprecia el resultado general de la investigación, considerando las cuatro dimensiones con un $85.4 \%$ en bienestar individual, un $86.2 \%$ en condiciones y medio ambiente de trabajo, con $85.4 \%$ en organización y un $84 \%$ en bienestar logrado a través del trabajo, con un resultado global de calidad de vida de un $85.26 \%$.

Tabla 3. Resultados por dimensión

\begin{tabular}{ll}
\hline \multicolumn{1}{c}{ Dimensión } & Resultado \\
\hline $\begin{array}{l}\text { Bienestar individual } \\
\text { Condiciones y medio ambiente } \\
\text { de trabajo }\end{array}$ & $85.4 \%$ \\
$\begin{array}{l}\text { Organización } \\
\text { Bienestar logrado a través del } \\
\text { trabajo }\end{array}$ & $84 \%$ \\
Total & $85.4 \%$ \\
\hline & Fuente: elaboración propia
\end{tabular}

Dentro de los resultados encontrados es importante mencionar que las Redes Neuronales Artificiales son un método que permiten resolver problemas, Generalidades de las redes neuronales artificiales 13 de forma individual o combinada con otros métodos, para aquellas tareas de clasificación, identificación, diagnóstico en las que el balance datos/conocimiento se inclina hacia los datos (Asanza, \& Olivo, 2018).

Además (Simon, 1999) encuentran que las ANN (Artificial Neural Networks) se aplica en campos tan diversos como el modelado, análisis de series temporales, reconocimiento de patrones, procesamiento de señales y control en virtud de una propiedad importante: la capacidad de aprender de los datos de entrada con o sin un maestro.

Estructura básica de una Red Neuronal Según (Olabe \& Basigain, 2001, pág. 2), la neurona se constituye como la unidad fundamental dentro del sistema nervioso, cada una de estas es una unidad procesadora que puede recibir y enviar señales a otras unidades, y así, si un sistema de entradas es fuerte se activa una salida especifica.

Tabla 4.

\section{Resumen de procesamiento de casos}

N Porcentaje

$\begin{array}{lll}\text { Entrenamiento } \quad 243 & 68.5 \%\end{array}$

\begin{tabular}{lcc}
\hline Pruebas & 112 & $31.5 \%$ \\
\hline Válido & 355 & $100.0 \%$ \\
\hline Excluido & 1 & \\
\hline Total & 356 & \\
\hline
\end{tabular}

En la construcción del modelo de redes neuronales, se utilizó una red neuronal, con arquitectura del tipo perceptrón multicapa de dos capas ocultas con función de activación Tangente hiperbólica y función de activación de la capa de salida Sigmoide. En el análisis de las variables se estandarizaron y se utilizó el software IBM SPSS Statistics 26. En la Figura 1 se muestra la estructura del modelo RNA diseñado. 


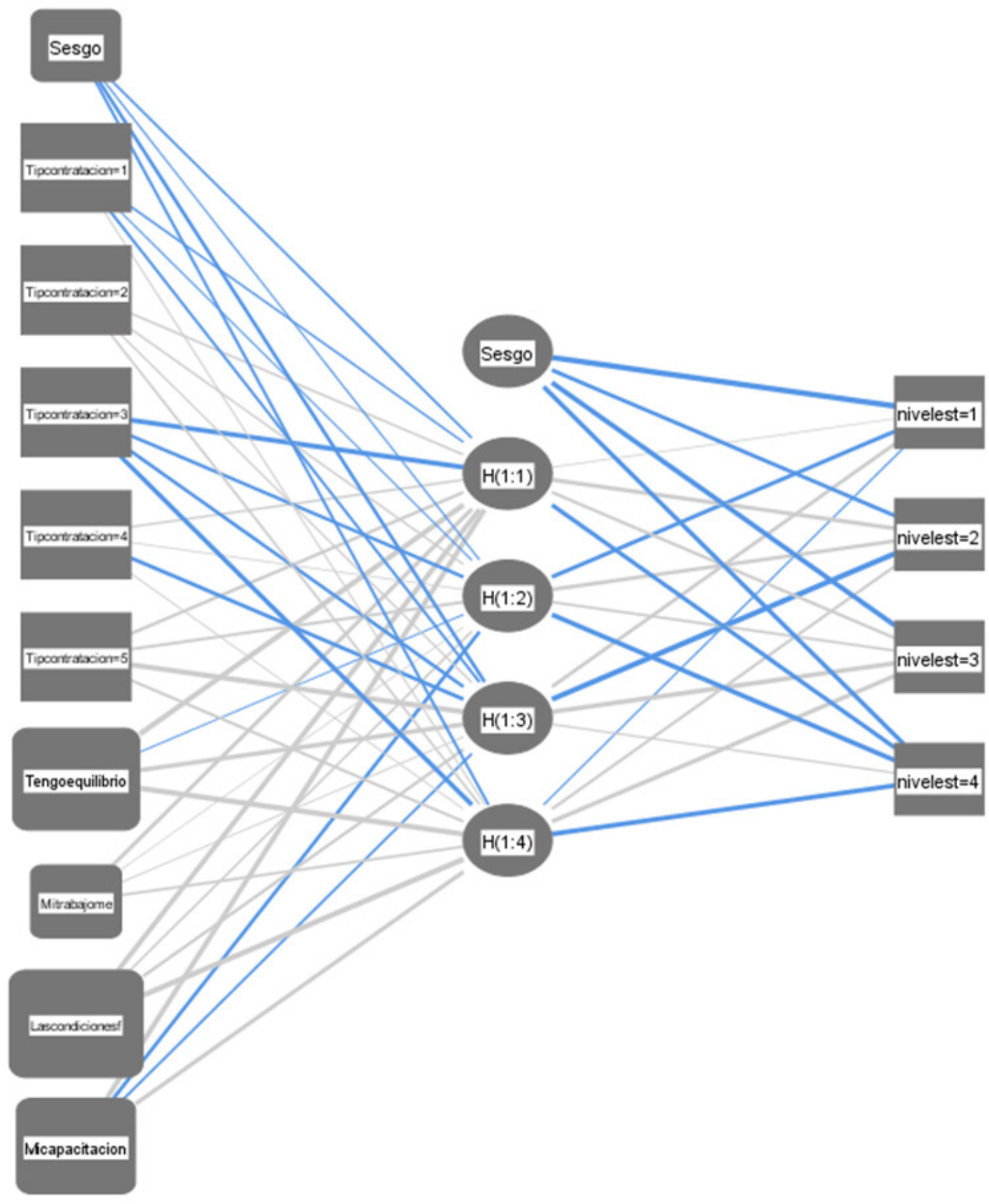

Función de activación de capa oculta: Tangente hiperbólica

Función de activación de capa de salida: Sigmoide

Figura 1. Red neuronal para el pronóstico de variables de calidad de vida laboral. 
El modelo seleccionado generó una precisión para pronosticar la pertenencia de las pequeñas y medianas, En la Tabla 5, se muestran los resultados de pronóstico de las muestras de entrenamiento y prueba. Donde la variable dependiente o de clasificación se expresa por el nivel de estudios, logrando un $87 \%$ como mejor evaluada en el nivel licenciatura.

Tabla 5: Pronóstico de pertenencia de clasificación

\begin{tabular}{|c|c|c|c|c|c|c|}
\hline \multirow[b]{2}{*}{ Muestra } & \multirow[b]{2}{*}{ Observado } & \multicolumn{5}{|c|}{ Pronosticado } \\
\hline & & \multicolumn{2}{|c|}{ Primaria Secundaria } & $\begin{array}{c}\text { Preparatoria o } \\
\text { técnica }\end{array}$ & Licenciatura & \multirow{2}{*}{$\begin{array}{r}\begin{array}{c}\text { Porcentaje } \\
\text { correcto }\end{array} \\
0.0 \%\end{array}$} \\
\hline \multirow{5}{*}{ Entrenamiento } & Primaria & 0 & 1 & 0 & 0 & \\
\hline & Secundaria & 0 & 90 & 6 & 18 & $78.9 \%$ \\
\hline & $\begin{array}{l}\text { Preparatoria o } \\
\text { técnica }\end{array}$ & 0 & 20 & 30 & 1 & $58.8 \%$ \\
\hline & Licenciatura & 0 & 9 & 1 & 67 & $87.0 \%$ \\
\hline & $\begin{array}{l}\text { Porcentaje } \\
\text { global }\end{array}$ & $0.0 \%$ & $49.4 \%$ & $15.2 \%$ & $35.4 \%$ & $77.0 \%$ \\
\hline \multirow{5}{*}{ Pruebas } & Primaria & 0 & 1 & 0 & 0 & $0.0 \%$ \\
\hline & Secundaria & 0 & 45 & 5 & 8 & $77.6 \%$ \\
\hline & $\begin{array}{l}\text { Preparatoria o } \\
\text { técnica }\end{array}$ & 0 & 6 & 11 & 3 & $55.0 \%$ \\
\hline & Licenciatura & 0 & 3 & 0 & 30 & $90.9 \%$ \\
\hline & $\begin{array}{l}\text { Porcentaje } \\
\text { global }\end{array}$ & $0.0 \%$ & $49.1 \%$ & $14.3 \%$ & $36.6 \%$ & $76.8 \%$ \\
\hline
\end{tabular}

Tabla 6. Importancia de las variables independientes

\begin{tabular}{lcr}
\hline \multicolumn{1}{c}{ Variables } & Importancia & $\begin{array}{c}\text { Importancia } \\
\text { normalizada }\end{array}$ \\
\hline Tipo de contratación & .183 & $73.2 \%$ \\
\hline Tengo equilibrio entre mi trabajo y mi vida personal & .231 & $92.4 \%$ \\
\hline Mi trabajo me permite desarrollo personal & .129 & $51.8 \%$ \\
\hline Las condiciones físicas del entorno de trabajo & .249 & $100.0 \%$ \\
\hline $\begin{array}{l}\text { Mi capacitación es la adecuada para el puesto de trabajo } \\
\text { que ocupo }\end{array}$ & .208 & $83.5 \%$ \\
\hline
\end{tabular}


En la tabla 6 se observa que las variables independientes más altas son las condiciones físicas del entorno con una importancia normalizada del $100 \%$.

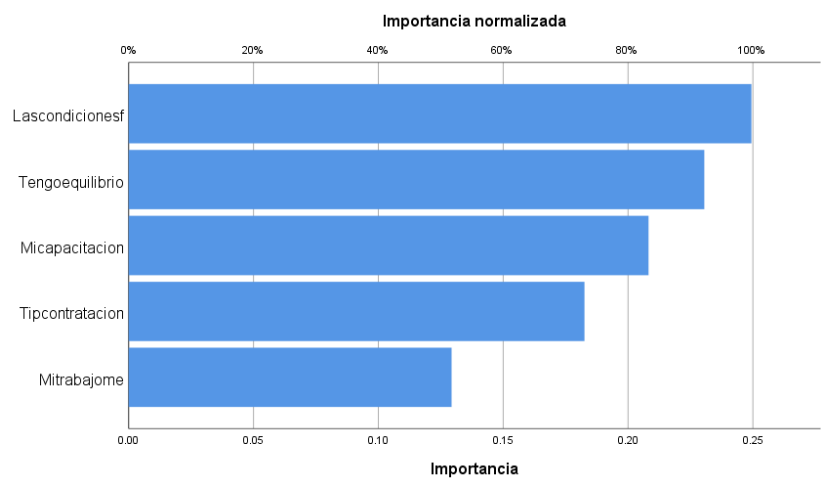

Figura 2. Importancia normalizada de las variables independientes

En la figura 2 se observa el orden de importancia normalizada de las variables independientes, ya que según un estudio realizado por Fontalvo et al (2018) aplico un modelo donde generó una precisión para pronosticar la pertenencia de las pequeñas empresas exportadoras del 100\% en la muestra, donde se evidencia empíricamente que su investigación aporta una estructura robusta para predecir el comportamiento de la eficiencia financiera en el sector objeto de esta investigación al observarse $94.44 \%$ de confiabilidad en la predicción global de la reserva.

\section{CONCLUSIONES}

El impacto inmediato del presente trabajo de investigación es que se puede aplicar la metodología a otras empresas a nivel nacional e Internacional. De acuerdo con Vaca (2015) al aplicar los programas de mejoramiento de calidad de vida laboral conlleva a grandes beneficios no solo para la empresa, sino también al trabajador. Los beneficios para la empresa conforman diferentes aspectos: Mejora el funcionamiento financiero, incrementa el valor del mercado, incrementa la productividad, reduce los costos operativos, mejora la habilidad para retener y atraer mejores empleados, fortalece la confianza y la lealtad de los empleados, reduce el ausentismo y fortalece la reputación. Al mejorar en estos aspectos la empresa y los trabajadores mejoran su desempeño laboral y su competitividad en el mercado, así como su productividad y su rentabilidad, es por ello que las empresas deben estar en constante actualización de acuerdo al programa de mejoramiento de calidad de vida laboral para brindar a sus trabajadores un ambiente sano de trabajo donde puedan crecer y sentir satisfacción por las actividades que realizan si el trabajador se siente a gusto en su trabajo la empresa genera más productividad por parte de ellos y mayores ganancias.

\section{REFERENCIAS}

1. Asanza, W. R., \& Olivo, B. M. (2018). Redes neuronales artificiales aplicadas al reconocimiento de patrones. Editorial UTMACH.

2. Cardona-Arias, J. A., \& Higuita-Gutiérrez, L. F. (2014). Aplicaciones de un instrumento diseñado por la OMS para la evaluación de la calidad de vida. Revista Cubana de Salud Pública, 40(2), 175-189.

3. Carrasquel, S. R. (2016). ¿Para qué se estudia la calidad de vida laboral? Revista interamericana de psicología ocupacional, 17(1), 34-53.

4. Juárez, A., \& Contreras, F. (2012). Calidad de vida y liderazgo: influencia de la calidad de vida percibida del directivo colombiano sobre sus prácticas de liderazgo.

5. Fontalvo, Tomás, De La Hoz, Efraín, \& De La Hoz, Enrique. (2018). Data Envelopment Analysis Method and Neural Networks in the Evaluation and Prediction of the Technical Efficiency of Small Exporting Companies. Información tecnológica, 29(6), 267-276.

6. González Alonso, J., \& Pazmiño Santacruz, M. (2015). Cálculo e interpretación del Alfa de Cronbach para el caso de validación de la consistencia interna de un cuestionario, con dos posibles escalas tipo Likert. Revista Publicando, 2(1), 62-67.

7. Granados, I. (2011). Calidad de vida laboral: historia, dimensiones y beneficios. Revista de 
Hinojosa

8. J. A. S., \& Rivas, J. G. D. (2009). La capacitación en las Pequeñas y Medianas Empresas (PYMES) de México.

9. Hernández Sampieri, R., Fernández Collado, C., \& Baptista Lucio, P. (2014). Metodología de la investigación (Vol. 3). México: McGraw-Hill.

10. Hernández-Vicente, I. A., Lumbreras-Guzmán, M., Méndez-Hernández, P., Rojas-Lima, E., Cervantes-Rodríguez, M., \& Juárez-Flores, C. A. (2017). Validación de una escala para medir la calidad de vida laboral en hospitales públicos de Tlaxcala. Salud-pública de México, 59, 183-192.

11. Huerta Riveros, P. C., Pedraja Rejas, L. M., Contreras Espinoza, S. E., \& Almodóvar Martínez, P. (2011). Calidad de vida laboral y su influencia sobre los resultados empresariales. Revista de Ciencias Sociales (Ve), 17(4).

12. Lizarazo, C., Fajardo, J., Berrio, S., \& Quintana, L. (2011). Breve historia de la salud ocupacional en Colombia. Archivos de prevención de riesgos laborales, 14(1), 38-42.

13. Manríquez, M. R., Salazar, A. L., \& Mateo, C. L. (2015). Ética y calidad laboral: impacto en el desempeño empresarial. Un estudio empírico. HOLOS, 3, 308-320.

14. Martínez Buelvas, L., Oviedo-Trespalacios, O., \& Luna Amaya, C. (2013). Condiciones de trabajo que impactan a la calidad de vida laboral. Revista Científica Salud Uninorte, 29(3).

15. Olabe, \& Basigain, X. (2001). REDES NEURONALES ARTIFICIALES Y SUS APLICACIONES. Escuela Superior de Ingeniería de Bilbao.

16. Simon, H. (1999). Neural Network. United States: Prentice-Hall, Inc.

17. Zapata, D. P., Montecinos, J. P., \& Jara, P. F. D. (2014). Influencia de variables organizacionales en la calidad de vida laboral de funcionarios del sector público de salud en el extremo norte de Chile. Universitas Psychologica, 13(2), 541-551.

18. Urzúa, A., \& Caqueo-Urízar, A. (2012). Calidad de vida: Una revisión teórica del concepto. Terapia psicológica, 30(1), 61-71.

19. Vaca Almeida, M. G. (2015). Diseño de un plan de acción para el mejoramiento del nivel de calidad de vida laboral (CVL) en los colaboradores de una empresa pública en el segundo período 2014 (Bachelor's thesis, PUCE). 